\title{
APROXIMACIÓN A UNA EXPERIENCIA REGIONAL DE RESPONSABILIDAD SOCIAL EMPRESARIAL EN EL SUROCCIDENTE COLOMBIANO
}

\section{APPROXIMATION OF A REGIONAL CORPORATE SOCIAL RESPONSIBILITY EXPERIENCE IN SOUTHWESTERN COLOMBIA}

\section{APROXIMAÇÃO DE UMA EXPERIÊNCIA RESPONSABILIDADE SOCIAL CORPORATIVA REGIONAL NO SUDOESTE DA COLÔMBIA}

\author{
Por: Andrés Ramiro Azuero-Rodríguez, Mónica García-Solarte, \\ Benjamín Betancourt-Guerrero
}

\begin{abstract}
Artículo de investigación producto del proyecto "Sistematización de la estrategia Región Socialmente Responsable en los municipios de Caloto, Guachené, Puerto Tejada y Villarrica” C.I 8099 Financiado por la Vicerectoria de Investigaciones de la Universidad del Valle.

Magíster en Administración, Universidad del Valle. Miembro del Grupo de Investigación Humanismo y Gestión. Profesor tiempo completo del departamento de Administración y Organizaciones, Facultad de Ciencias de la Administración, Universidad del Valle. Email: andres.azuero@correounivalle.edu.co, Colombia.

Master of Management de la Universidad de Tulane. Miembro del Grupo de Investigación Humanismo y Gestión. Profesora tiempo completo del departamento de Administración y Organizaciones, Facultad de Ciencias de la Administración, Universidad del Valle.Email: monica.garcia@correounivalle.edu.co, Colombia. Magíster en Administración, Universidad del Valle. Miembro del Grupo de Investigación Previsión y Pensamiento Estratégico. Profesor tiempo completo del departamento de Administración y Organizaciones, Facultad de Ciencias de la Administración, Universidad del Valle. Email: benbenjamin.betancourt@ correounivalle.edu.co, Colombia.
\end{abstract}

Recibido: 02 de junio de 2015

Aprobación definitiva: 15 de octubre de 2015

\section{Resumen}

Hoy es importante la articulación de diversos actores en torno a experiencias de responsabilidad social regional, que aporten al desarrollo local, dadas las múltiples necesidades de las regiones que no pueden ser resueltas por un solo actor. Esta articulación es una oportunidad de construir proyectos desde múltiples perspectivas y capacidades locales. Por ello, los grupos de investigación Humanismo y Gestión y Previsión y Pensamiento Estratégico de la Universidad del Valle, plantean una investigación que tiene como objetivo identificar y visibilizar los avances, logros, aprendizajes, dificultades y retos del proceso de 
consolidación de la experiencia de región socialmente responsable en el norte del Cauca. Para ello se realiza un estudio de tipo exploratorio, descriptivo con enfoque cualitativo donde se emplean técnicas como entrevistas en profundidad, grupos focales y talleres de formulación colectiva con los actores de la región. Los resultados muestran que la estrategia ha aportado a la visibilización de la región y a la construcción de proyectos conjuntos de mayor impacto contando con la participación de empresarios, instituciones y gobierno. Se reconocen dificultades como la de lograr acuerdos institucionales en torno algunos temas y la consecución y disposición de recursos por parte del gobierno nacional, para resolver problemas locales. Esta experiencia permite reflexionar sobre aprendizajes, retos y dificultades que aunque son particulares pueden ser un ejemplo para otras regiones que estén interesadas en articularse en torno a proyectos conjuntos.

Palabras claves: Responsabilidad Social Empresarial, Región Socialmente Responsable, Norte del Cauca.

JEL: M14, M19

\section{ABSTRACT}

Today is important articulation of different actors around regional social responsibility experiences that contribute to local development, given the multiple needs of the regions that can not be resolved by a single actor. This articulation is an opportunity to build projects from multiple perspectives and local capacities. Therefore, research groups and Humanism Management and Security and Strategic Thinking of the Universidad del Valle, pose a research that aims to identify and make visible progress, achievements, lessons learned, difficulties and challenges in the consolidation of the experience of region socially responsible in northern Cauca. To do an exploratory and descriptive qualitative approach where techniques are used as in-depth interviews, focus groups and workshops with the actors collective development of the region is performed. The results show that the strategy has contributed to the visibility of the region and the construction of joint projects with the greatest impact with the participation of entrepreneurs, institutions and government. Difficulties as to achieve institutional agreements on some issues and procurement and provision of funds by the national government are recognized, to solve local problems. This allows for reflection on learning experiences, challenges and difficulties but they are individuals can be an example for other regions who are interested in joint around joint projects.

Key words: Corporate Social Responsibility, Socially Responsible Region, Northern Cauca.

JEL: M14, M19

\section{RESUMO}

Hoje é importante articulação de diferentes atores em torno de experiências de responsabilidade social regionais que contribuam para o desenvolvimento local, tendo em conta as múltiplas necessidades das regiões que não podem ser resolvidos por um único ator. Essa articulação é uma oportunidade para construir 
Andrés Ramiro Azuero-Rodríguez, Mónica García-Solarte, Benjamín Betancourt-Guerrero Aproximación a una experiencia regional de responsabilidad social empresarial en el suroccidente colombiano

projetos de múltiplas perspectivas e capacidades locais. Por isso, grupos de pesquisa e Gestão e Segurança e Pensamento Estratégico da Universidad del Valle Humanismo, representam uma pesquisa que tem como objetivo identificar e fazer progressos visíveis, realizações, lições aprendidas, dificuldades e desafios na consolidação da experiência da região socialmente responsável no norte do Cauca. Para fazer uma abordagem qualitativa exploratória e descritiva, onde são utilizadas técnicas como entrevistas em profundidade, grupos focais e oficinas com os atores desenvolvimento coletivo da região é realizado. Os resultados mostram que a estratégia tem contribuído para a visibilidade da região ea construção de projetos conjuntos com o maior impacto com a participação de empresários, instituições e governo. Dificuldades como para alcançar acordos institucionais sobre algumas questões e aquisição e fornecimento de fundos pelo governo nacional são reconhecidos, para resolver problemas locais. Isto permite a reflexão sobre experiências de aprendizagem, desafios e dificuldades, mas eles são os indivíduos podem ser um exemplo para outras regiões que estão interessados em conjunto em projectos conjuntos

Palavras-chave: Responsabilidade Social Corporativa, Socialmente Responsável Region, Northern Cauca.

JEL: M14, M19

\section{Introducción}

La Responsabilidad Social Empresarial (RSE) hace parte en la actualidad de la agenda de la mayoría de las empresas, donde se identifica que la noción se ha transformado de prácticas de filantropía a un enfoque estratégico que se fundamenta en el desarrollo y beneficio interdependiente de los grupos de interés. Adicionalmente, hoy el conjunto de empresas que comparten espacios geográficos, se unen para realizar propuestas de Responsabilidad Social de alcance regional, siendo un ejemplo de ello la región del norte del Cauca, que en beneficio de las normas del país propone un desarrollo que redunde en la región. Es por ello que a partir de la investigación realizada desde los grupos de investigación Humanismo y Gestión y Previsión y Pensamiento Estratégico de la Facultad de Ciencias de la Administración de la Universidad del Valle, se proponen como objetivo identificar y visibilizar los logros, avances, aprendizajes, dificultades y retos del proceso de consolidación de la estrategia región socialmente responsable en el norte del Cauca. Para ello se desarrolla un estudio cualitativo, en el que se sistematiza la experiencia vivida en el norte del Cauca desde la voz de los actores involucrados en el proceso, a través de entrevistas y grupos focales de empresarios, sector educativo, organizaciones públicas y cívicas de la región. Los resultados muestran que el avance mas significativo es el incremento del empleo y el proceso de generación de confianza entre los actores de la región, el logro que mas se aprecia es la intervención por parte del gobierno nacional, la presencia del sector educativo y la visibilidad de la región a 
nivel nacional, con relación a los aprendizajes es el empoderamiento de lideres de la región y la formación en temas de RSE, reconocimiento que para generar cambio se requiere del compromiso de todos los actores, no se desarrolla de manera unilateral, la principal falla es el retraso de alianzas en las instituciones, falta de liderazgo, dificultad para conciliar todos los intereses, falta de reconocimiento de algunos actores involucrados, disponibilidad de recursos. Entre los principales retos planteados por las actores consultados están el cambio generacional, la continuidad misma del proyecto, la articulación adecuada empresa, gobierno, sociedad y academia, el posconflicto, la articulación con las comunidades indígenas y afro, la relación gobierno empresa, la visión compartida del futuro, y la medición de los impactos de la región socialmente responsable. La principal conclusión, a pesar que los actores reconocen que aún hay errores y retos en el proceso y algunos acuerdos siguen siendo incipientes, es que se han logrado avances en educación agua potable, pobreza y empleo.

\section{Marco Teórico}

Dada la diversidad de enfoques y aproximaciones al tema de la Responsabilidad Social Empresarial (RSE) (Wartick y Mahon, 1994; Carroll, 1999; Garriga y Melé, 2004; Porter y Kramer, 2002; Van Tulder y Van der Zwart 2006, Raufflet, 2011), su relación con el desarrollo local en un territorio y una metodología acorde a la necesidad de registrar dicho proceso en términos cuantitativos y cualitativos, para efectos de este proyecto se presenta a continuación la forma de concebir dichos conceptos para la realización del análisis y reflexión del mismo.

\section{Responsabilidad social empresarial}

La Responsabilidad Social Empresarial (RSE) como concepto ha tenido un recorrido desde la segunda mitad del siglo XX dentro de la literatura empresarial, y es desde 1950 que se empieza a distinguir el término dentro de la concepción capitalista (Crespo, 2010), y se dan ideas vagas respecto a lo que la RSE significa. En la evulución del concepto en los años 50's se hace énfasis en hechos filantrópicos o de voluntariados (Carroll, 1999; Bowen, 1953), mientras que a partir de los años 70's el concepto emprende un camino de asociación o correspondencia entre las empresas y la sociedad (Steiner, 1971), por otro lado, Friedman (1970) menciona que las empresas solo tienen como responsabilidad social el incremento de sus ganancias, y que deben hacerlo desde una competencia sana, sin engaños, sin fraude, incorporándose a las leyes y normas de la sociedad a la cual pertenece la organización.

De acuerdo con Crespo (2010) a partir de los años 80's los estudios acerca de la RSE incorpora elementos empiricos, lo cual implicaba una mirada más profunda en las prácticas de las organizaciones enfocadas hacía la sociedad. Es asi como Drucker (1984) propone que la RSE se vuelve insumo y resultado para transformar una problemática social en un aprovechamiento economico, lo cual implica ganancias, productividad, ventajas competitivas, entre otros. En los 90's, el concepto de RSE avanza hasta asociarse con la teoría de los Stakeholders o ciu- 
Andrés Ramiro Azuero-Rodríguez, Mónica García-Solarte, Benjamín Betancourt-Guerrero

Aproximación a una experiencia regional de responsabilidad social empresarial en el suroccidente colombiano

dadanía corporativa y la teoría de ética empresarial (Crespo, 2010, p. 123). Es así como Mercado y García (2007) plantean que la RSE presenta varias dimensiones y que por tanto, las organizaciones no limitan sus acciones al público interno (a sus colaboradores y accionistas), sino que involucra otros públicos, tales como: clientes, los proveedores, el medio ambiente, el gobierno y la sociedad en general, y de acuerdo a Mark y von Schantz (2007) las organizaciones trabajan por la RSE desde las expectativas de sus grupos de interés.

A partir del Siglo XXI, el planteamiento de Porter y Kramer, (2002) abarca la RSE como pieza trascendental en el direccionamiento estratégico de las organizaciones, dada su implicación directa en los resultados y en el mejoramiento de la percepción de la imagen corporativa y su reputación. Por su parte Guerra (2007), abarca la comprensión de la RSE desde el ámbito de la gestión estratégica y el impacto social de la actividad específica de las organizaciones con el entorno, comprendiendo "el valor social, subjetivo y cualitativo como complemento de los criterios tradicionales de medición y evaluación de la rentabilidad y gestión financiera, en su vinculación e interacción con el medio o entorno” (Guerra, 2007: 77).

Por otra parte, Correa, Flynn y Amit (2004) citado por Montes (2008) exponen tres propuestas de la visión moderna de la RSE desde instituciones que sugieren las siguientes definiciones:

WBCSD de Suiza: "La RSE con un enfoque ético, es decir, se presenta como un compromiso asumido por la empresa con la colaboración de sus empleados, sus familias, la comunidad local y la sociedad".

Prince of Wales Business Leadership Forum (PWBLF), de Inglaterra: "Asume la RSE desde el punto de vista positivo, como el conjunto de prácticas empresariales basadas en el respeto hacia los empleados, las comunidades y el ambiente".

Business for Social Responsibility (BSR), de Estados Unidos: "Presenta un enfoque normativo, define la RSE como la administración de un negocio de forma que cumpla o sobrepase las expectativas éticas, legales, comerciales y públicas".

Siguiendo la línea de la concepción de la RSE, de acuerdo a Barroso (2008), la definición sugerida por el Centro Mexicano para la Filantropía (CEMEEFI) es la que más se acerca a una concepción empírica; ésta dice que la RSE comprende el "compromiso consciente y congruente de cumplir tanto en lo interno como en lo externo" con las expectativas de todos sus stakeholders para construir el bien común y una mejor sociedad.

\section{RSE en Colombia}

En Colombia varios autores han investigado sobre lo que se entiende por RSE, en el cual existe una larga trayectoria de participación del empresariado en iniciativas con impacto en la sociedad. Según Gutiérrez, Avella y Villar (2006) la participación ha evolucionado de esfuerzos caritativos a inversión social y, en los últimos 
años, a filantropía estratégica. De acuerdo con estos autores en Colombia hay por lo menos cuatro modelos diferentes de inversión de las empresas en la sociedad:

1) Creación de programas particulares al interior de la empresa;

2) Creación de organizaciones fuera de la empresa, como fundaciones, corporaciones o asociaciones, o apoyo a organizaciones sin fines de lucro ya existentes;

3) Participación en programas sociales liderados por su gremio; y

4) Participación en programas del gobierno de turno.

Estos modelos se acompañan de la conformación conceptual de instancias del sector productivo colombiano tales como el Comité y la Gerencia de Responsabilidad Social que en la actualidad reside en la Gerencia de Arquitectura Social Estratégica de la ANDI , y de la creación de organizaciones dedicadas a promover la Responsabilidad Social, tales como el Consejo Empresarial Colombiano para el Desarrollo Sostenible CECODES, creado en 1993, conformado por 38 empresas y 4 gremios de los sectores más relevantes para la economía colombiana (agroindustria, minería, forestal, energía, manufacturera, construcción y financiera). Desde el sector financiero Asobancaria es la entidad que agremia y promueve proyectos desde la banca, además es quien divulga información relevante para todos los grupos que inciden directa o indirectamente en la gestión de las organizaciones que pertenecen al sector. En la tabla No. 1 se puede apreciar algunas de las definiciones del tema en organizaciones relevantes para el país.

Desde el planteamiento de Giraldo (2011) en un estudio realizado en organizaciones antioqueñas propone que el enfoque de la RSE debe darse desde diferentes ámbitos complementarios entre los cuales están: la retribución a la sociedad, apoyo a la comunidad de sus alrededores, ayuda a sus colaboradores, cumplimiento de la normatividad del país, compromiso ético en los negocios, compromiso proactivo frente a los clientes, y generación de ganancias y utilidades y asimismo beneficios económicos. Por otro lado, de acuerdo a Crespo (2010), el tópico de RSE se desarrolla desde dos marcos de referencia complementarios: el primero comprende la transformación del concepto desde el campo teórico, relacionado con el contexto empresarial y su implicación con la problemática social; y, por otro lado, comprende las prácticas de RSE estudiadas desde dentro y no por fuera de ellas, relacionando lo teórico con lo empírico.

De acuerdo con Fajardo (2010), la actual visión de la RSE en Colombia presenta "grandes desequilibrios sociales, una exclusión social marcada, indicadores de concentración de ingresos en unos pocos, niveles de desocupación altos, carencia de espacios para el diálogo, y aumento de las tasas de miseria e informalidad", lo cual constituye argumentos para que el sector privado, en especial los dueños, directivos y gerentes se organicen y emprendan acciones comprometidas a nivel institucional frente a la RSE. 
Andrés Ramiro Azuero-Rodríguez, Mónica García-Solarte, Benjamín Betancourt-Guerrero

Aproximación a una experiencia regional de responsabilidad social empresarial en el suroccidente colombiano

Tabla No. 1. Definiciones de RSE

\begin{tabular}{|l|l|}
\hline $\begin{array}{l}\text { Asociación Nacional } \\
\text { de Industriales (ANDI) } \\
\text { Colombia - Gerencia } \\
\text { de Arquitectura Social } \\
\text { Estratégica }\end{array}$ & $\begin{array}{l}\text { "Respuesta que la empresa debe dar a las expectativas en los } \\
\text { sectores con los cuales ella tiene relación, en materia de desarrollo } \\
\text { integral de sus trabajadores y en el aporte a la comunidad que le } \\
\text { permitió crecer y desarrollarse". }\end{array}$ \\
\hline $\begin{array}{l}\text { ISO (Organización In- } \\
\text { ternacional de Norma- } \\
\text { lización) - ISO 26000 }\end{array}$ & $\begin{array}{l}\text { "Responsabilidad ante los impactos de sus decisiones y actividades } \\
\text { en la sociedad y el medio ambiente, a través de un comporta- } \\
\text { miento transparente y ético que sea: consistente con el desarrollo } \\
\text { sostenible y el bienestar general de la sociedad; considere las } \\
\text { expectativas de sus partes interesadas; esté en cumplimiento } \\
\text { con la legislación aplicable y sea consistente con normas inter- } \\
\text { nacionales de comportamiento; y esté integrada a través de toda } \\
\text { la organización". }\end{array}$ \\
\hline $\begin{array}{l}\text { Centro Colombiano } \\
\text { de Responsabilidad } \\
\text { Empresarial -CCRE- }\end{array}$ & $\begin{array}{l}\text { "La Responsabilidad Social es la capacidad de respuesta que tiene } \\
\text { una empresa o una entidad, frente a los efectos e implicaciones de } \\
\text { sus acciones sobre los diferentes grupos con los que se relaciona } \\
\text { (stakeholders o grupos de interés). De esta forma las empresas } \\
\text { son socialmente responsables cuando las actividades que realiza } \\
\text { se orientan a la satisfacción de las necesidades y expectativas de } \\
\text { sus miembros, de la sociedad y de quienes se benefician de su } \\
\text { actividad comercial, así como también, al cuidado y preservación } \\
\text { del entorno". }\end{array}$ \\
\hline $\begin{array}{l}\text { Corporación Fenalco } \\
\text { Solidario }\end{array}$ & $\begin{array}{l}\text { "Acciones voluntarias que hacen las organizaciones para contribuir } \\
\text { a la sostenibilidad del planeta, es decir, buscar que las organiza- } \\
\text { ciones no se queden en el ámbito legal y obligatorio, sino que } \\
\text { trasciendan y contribuyan en el ámbito social, con la comunidad } \\
\text { y con sus grupos de interés. La responsabilidad social está orien- } \\
\text { tada al negocio, es estratégica con todos esos grupos de interés". }\end{array}$ \\
\hline
\end{tabular}

Fuente: Elaboración Propia.

\section{Región socialmente responsable}

El reconocimiento del impacto que tienen las empresas en el territorio donde operan, implica el tener en cuenta los alcances de sus actuaciones en los grupos de interés y las posibilidades de actuación en términos de formas de articulación con otros actores que le apuesten al desarrollo local, tanto económico, social y ambientalmente hablando.

Alburquerque (2003) plantea que el panorama para el siglo XXI converge hacía mayores cambios en la tecnología, en la comunicación, en la estructuración de una economía fortalecida por las instituciones, y el involucramiento del tejido social en el desarrollo de innovación, de competencias, y de esta manera buscar el fortalecimiento local o territorial. 
Parte del desarrollo temático de "Región socialmente responsable" se encuentra en el desglose de los conceptos intrínsecos, siendo Región uno de ellos; este es definido por Flórez, Neira, y Osorio (2005), quienes proponen que región es el "espacio no solamente geográfico o ambiental", sino que incluye diversos sentidos y significados unidos a la apropiación del territorio desde su ámbito social, cultural y económico, además de tener como referente la dimensión local, nacional y global. Desde el planteamiento de Villacorta (2008), el concepto de región está articulado a los miembros que la integran, el compromiso entre las instituciones que la conforman, las asociaciones entre lo público y lo privado, y la integridad que fortalece el nivel de confianza en la sociedad y la autoridad gubernamental, enfocado a la calidad de vida de la población de la región involucrada. Asimismo, región desde el planteamiento de Azuero, Melo y García, 2011: 115, se entiende como un "sistema interdependiente de relaciones colaborativas y de compromisos mutuos con una visión sostenible del territorio, que se garantiza a través de la construcción de redes de confianza y reciprocidad”.

Por otro lado, desde la perspectiva de Pamplona (2014), existe un concepto que se acerca a la concepción de región socialmente responsable, el cual es denominado como Estrategia de Región; de acuerdo al autor esta última comprende acciones encaminadas a impulsar el desarrollo desde la dimensión económica, política, social y ambiental de un territorio; adicionalmente menciona una propuesta realizada en la zona de Antioquia, que tiene como base los aspectos mencionados; la iniciativa se denominó "La nueva energía paisa”, la cual converge hacía el desarrollo local de la zona conocida como el Valle de San Nicolás:

La nueva energía paisa es una estrategia de región que impulsa lo local, en la medida en que ubica en el centro del proceso de desarrollo a los actores y a una serie de factores locales que impulsan el desempeño económico, social, político y medioambiental (Rosales \& Chauca, 2010: 11).

Ahora bien, en el campo del Desarrollo Local, se conjugan aspectos que convergen hacía el desarrollo del concepto de Región Socialmente Responsable; de acuerdo con Rosales \& Chauca (2010), el desarrollo local no abarca únicamente el crecimiento de indicadores o cifras locales, sino que se comprende la dimensión humana y social, el desarrollo sostenible, la competitividad y la geografía. Desde el punto de vista de Alburquerque (2003) el desarrollo local se integra como estrategia al desarrollo macroeconomico de una región, orientando acciones que introducen la innovación tecnológica, de gestión, social e institucional en los diferentes sistemas productivos locales y organizaciones. Asimismo, Elizalde (2003) en el documento presentado por la Dirección del Instituto Latinoamericano y del Caribe de Planificación Económica y Social (ILPES) denominado "Planificación estratégica territorial y políticas públicas para el desarrollo local” plantean que:

El desarrollo local se logra mediante un fomento productivo responsable y el fortalecimiento de la sociedad. A estos elementos, hay que sumar los aspectos ambientales para alcanzar un desarrollo sostenible (Elizalde, 2003: 8). 
Andrés Ramiro Azuero-Rodríguez, Mónica García-Solarte, Benjamín Betancourt-Guerrero

Aproximación a una experiencia regional de responsabilidad social empresarial en el suroccidente colombiano

Adicionalmente, Villar (2004) plantea que el desarrollo local puede observarse desde la implementación de un proyecto sostenible que contenga todas aquellas capacidades a nivel territorial (ambientales, sociales, técnicas, económicas, institucionales, entre otras) en pro del desarrollo sostenible. Por su parte, Tecco y Lucca (1998) plantean que el cambio en el entorno económico, político y social de las instituciones tanto públicas como privadas, propician la cooperación para el trabajo conjunto en pro del desarrollo local. Desde otra perspectiva:

[...] Una estrategia de desarrollo local puede iniciarse a partir de la coordinación territorial de algunos programas e instrumentos de fomento sectoriales definidos desde el nivel central pero que deben ser ejecutados territorialmente (Alburquerque, 2003: 16).

Por ende, teniendo en cuenta el planteamiento de Alburquerque (2003) todas las propuestas para garantizar el desarrollo a nivel económico, social y ambiental en una región, se deben regir por acuerdos tanto a nivel político como a nivel social en el ámbito territorial a que dé lugar.

Azuero, Melo y García (2011), exponen como la RSE se encuentra intrínseca al concepto de Región Socialmente Responsable organizado por el Global Compact, donde se menciona que las acciones llevadas a cabo por las organizaciones, instituciones y la sociedad que son parte de un territorio específico se conjugan en su sistema económico, político, social y cultural para garantizar un desarrollo sostenible.

Una región responsable implica considerar todas las dimensiones de la sociedad como son: la economía, la axiología, lo social, la cultura, la política, las relaciones y su impacto en el desarrollo humano (Salinas, 2008) y tiene como punto de partida la actividad productiva como motor del desarrollo en un modelo de trabajo que articula diversos actores tales como el sector productivo, la academia, las organizaciones sociales, la comunidad, el sector público, entre otros.

En otras experiencias por fuera del país, puede verse que en España existe una red denominada RETOS $^{\mathbf{1}}$ (Red de territorios socialmente responsables), el cual hace parte del Ministerio de Empleo y Seguridad Social de dicho país; de ésta hacen parte diferentes municipios, organizaciones y provincias integradas en red, con el fin mismo de articularse como estrategia para el desarrollo y el mejoramiento de la calidad de vida de la sociedad de los territorios que la constituyen.

En Colombia se destacan diferentes propuestas para el desarrollo de diferentes regiones partiendo de la cooperación, unión y la creación de alianzas públicasprivadas, entre las cuales "Norte del Cauca, Región Socialmente Responsable" se define desde el 2008 como una iniciativa que articula a la sociedad, organizacio-

1. EMPLEO Y SEGURIDAD SOCIAL (2012). Informe Buenas Prácticas RED RETOS: Análisis y validación de Buenas prácticas tomado el 30 de Abril de 2015 de http://www.empleo.gob.es/redretos/ es/contenidos/buenaspracticas/informes/Informe_Buenas_Practicas_Retos.pdf 
nes ubicadas en la región y las entidades gubernamentales, todo con la misión de disminuir la pobreza (objetivo del Pacto Mundial) y garantizar el desarrollo local.

\section{Región socialmente responsable en el norte del Cauca}

Durante 2008 el Global Compact impulsó en siete regiones colombianas la puesta en marcha de la estrategia Región Socialmente Responsable con el objetivo de generar mecanismos para el desarrollo local y regional. Para iniciar se realizó un Diplomado denominado "Empresas y Derechos Humanos" dirigido a los empresarios y coordinado por la Organización de las Naciones Unidas, y en asocio con agencias de cooperación, ofrecieron a empresarios del país el Diplomado Empresas y Derechos Humanos.

En el norte del Cauca la iniciativa de Región Responsable se desarrolla a partir de la decisión de algunos empresarios que identifican retos y condiciones sociales que les son comunes a las empresas, tales como la pobreza, la exclusión social, la falta de oportunidades entre otros, que fueron diagnosticados en diversas investigaciones (Roa, 2000; García 2007; Alonso, 2006; Aloson y Lotero, 2006; Fundación Carvajal 2007; CIDSE, 2009; PNUD 2009).

En el año 2010, La Universidad del Valle a través del Instituto de Prospectiva, Innovación y Gestión del Conocimiento, cofinanciado por Colciencias, desarrolló un estudio de prospectiva para la Región Responsable Norte del Cauca, donde se realizó una caracterización de la región y un plan de acción de acuerdo con los resultados de las consultas con los diferentes actores sociales de la región. El estudio de prospectiva permitió definir el escenario que todos los actores consultados encuentran como el deseable y viable para la región. Una sugerencia de este proyecto es la construcción de alianzas público privadas para el desarrollo de la región.

La revisión de estas investigaciones adelantadas en la zona permite encontrar como elementos comunes la problemática social y económica y los diversos grados de desarticulación de los actores en la región (comunidad, gobierno, empresarios, instituciones) a pesar del notable aumento de indicadores de crecimiento económico. Este hecho impone como uno de los principales retos el establecimiento de una agenda de trabajo articulado y la concertación de un modelo de desarrollo que involucre las diferentes dimensiones de la vida en la región. En ese sentido la Región Socialmente Responsable del Norte del Cauca se convierte en un espacio de encuentro y de oportunidad para la participación de los actores de la región.

\section{Metodología}

La investigación realizada es de tipo exploratoria, descriptiva y con un enfoque cualitativo. Su principal objetivo es la descripción y análisis de las características de un proceso para identificar el cómo se desarrolló la estrategia de Región Socialmente Responsable Norte del Cauca. 
Andrés Ramiro Azuero-Rodríguez, Mónica García-Solarte, Benjamín Betancourt-Guerrero

Aproximación a una experiencia regional de responsabilidad social empresarial en el suroccidente colombiano

Se realiza como una sistematización que permite una recuperación de los procesos, prácticas, lecciones, y percepciones de los actores involucrados en la experiencia. El tipo descriptivo se relaciona con las condiciones existentes, prácticas, opiniones de las personas, puntos de vista, actividades en marcha y resultados del proceso.

Para identificar los principales logros, avances, aprendizajes, dificultades y retos derivados de la estrategia Región Socialmente Responsable Norte del Cauca, se llevaron a cabo entrevistas individuales, grupos focales y talleres de formulación colectiva de las lecciones aprendidas, con los actores que se encontraban relacionados de manera directa o indirecta con la estrategia. Para el propósito de la experiencia, se identificaron las siguientes categorías:

- Logro: Resultado concreto del desarrollo de la experiencia.

- Avance: Situaciones que han mejorado comparadas con el estado inicial de la experiencia.

- Aprendizaje: conocimiento adquirido en el desarrollo de la experiencia.

- Dificultades: Obstáculos en el desarrollo de la experiencia.

- Retos: Desafíos a futuro en el desarrollo de la experiencia.

El trabajo de investigación se realizó en cuatro etapas de la siguiente forma: Etapa I: Análisis Documental sobre el tema y experiencias de aplicación. Etapa 2: Entrevista actores empresariales para identificar su posición sobre el tema y su relación con la región. Etapa 3: Consulta a actores relacionados con la experiencia a través de grupos focales y talleres de formulación colectiva. Etapa 4. Sistematización de los resultados.

En la Tabla No. 2 se identifican las técnicas utilizadas en las diferentes etapas.

En la etapa de consulta a los actores de la región, se realizaron actividades con 23 diferentes representantes de organizaciones que aceptaron participar del proyecto. La consulta se realizó en tres grandes grupos: 1) actor empresarial (empresas y fundaciones de la Zona Franca del Cauca), Cámara de Comercio seccional Santander de Quilichao y Asociación Nacional de Industrial ANDI Cauca, 2) actor gubernamental (gobiernos locales, representación del gobierno nacional), 3) actor educativo (entidades de formación a nivel técnico, tecnológico, profesional). Por ser un estudio de tipo cualitativo, no se diseñan muestras, ya que el propósito no son las generalizaciones, sino la descripción de actores del proceso (Mayan, 2009; Méndez, 2006). No hay un perfil específico y se considera como criterio de inclusión de los participantes, el ser directivos de organizaciones privadas, públicas o del tercer sector que operan en la zona y han estado vinculadas de forma directa o indirecta con la experiencia Región Socialmente Responsable Norte del Cauca, siguiente la propuesta de Willig (2001), sobre la selección de participantes en investigación cualitativa. 


\section{Resultados y Discusión}

Para lograr el objetivo del proyecto de identificar los principales logros, avances, aprendizajes, dificultades y retos derivados de la estrategia Región Socialmente Responsable Norte del Cauca, primero se identificaron y caracterizaron los actores que participaron en el desarrollo de la Región socialmente Responsable y las principales líneas de actuación de la estrategia.

Tabla No. 2. Técnicas de investigación utilizadas

\begin{tabular}{|c|c|c|}
\hline \multicolumn{2}{|c|}{ TÉCNICAS } & INSTRUMENTO \\
\hline \multicolumn{2}{|l|}{ 1. Análisis Documental } & \multirow[b]{2}{*}{$\begin{array}{l}\text { Matriz de } \\
\text { Lectura }\end{array}$} \\
\hline $\begin{array}{l}\text { Temas: } \\
\text { - Definiciones de Responsabili- } \\
\text { dad Social Empresarial RSE } \\
\text { - Región Socialmente Respon- } \\
\text { sable. } \\
\text { - Prácticas de RSE reportadas } \\
\text { por empresas de la región. }\end{array}$ & $\begin{array}{l}\text { Fuentes: } \\
\text { - Publicaciones académicas (electró- } \\
\text { nicas y físicas) orientadas al estudio } \\
\text { sobre la RSE. } \\
\text { - Documentos especializados y memo- } \\
\text { rias de eventos sobre RSE y experien- } \\
\text { cias regionales de RSE. } \\
\text { - Memorias de sostenibilidad y reportes } \\
\text { públicos de organizaciones del sector } \\
\text { productivo de la región. }\end{array}$ & \\
\hline \multicolumn{2}{|c|}{ 2. Entrevista con actores empresariales de la región } & \multirow[b]{2}{*}{$\begin{array}{l}\text { Guías de } \\
\text { Entrevista }\end{array}$} \\
\hline $\begin{array}{l}\text { Temas: } \\
\text { - Información de la Organiza- } \\
\text { ción. } \\
\text { - La Organización y la RSE. } \\
\text { - Prácticas de responsabilidad } \\
\text { social. } \\
\text { - Relación de la Organización } \\
\text { con la región. }\end{array}$ & $\begin{array}{l}\text { Fuentes: } \\
\text { Gerentes de Empresas de la Región } \\
\text { Dirección Camára de Comercio } \\
\text { Dirección ANDI Seccional } \\
\text { Gerente Zona Franca del Cauca }\end{array}$ & \\
\hline \multicolumn{2}{|c|}{ 3. Grupos Focales y Talleres de Formulación } & \multirow[b]{2}{*}{$\begin{array}{c}\text { Guía de } \\
\text { Grupo Focal }\end{array}$} \\
\hline $\begin{array}{l}\text { Temas: } \\
\text { - Región Norte del Cauca } \\
\text { - Caracterización de la expe- } \\
\text { riencia. } \\
\text { - Principales logros, avances, } \\
\text { aprendizajes, dificultades y } \\
\text { retos derivados de la estrate- } \\
\text { gia Región Socialmente Res- } \\
\text { ponsable Norte del Cauca. }\end{array}$ & $\begin{array}{l}\text { Fuentes: } \\
\text { 1) Actor empresarial (empresas y } \\
\text { fundaciones de la Zona Franca } \\
\text { del Cauca), Cámara de Comercio } \\
\text { seccional Santander de Quilichao } \\
\text { y Asociación Nacional de Industrial } \\
\text { ANDI Cauca. } \\
\text { 2) Actor gubernamental (gobiernos } \\
\text { locales, representación del gobier- } \\
\text { no nacional). } \\
\text { 3) Actor educativo (entidades de for- } \\
\text { mación a nivel técnico, tecnológico, } \\
\text { profesional). }\end{array}$ & \\
\hline
\end{tabular}

Fuente: Elaboración Propia 
Andrés Ramiro Azuero-Rodríguez, Mónica García-Solarte, Benjamín Betancourt-Guerrero

Aproximación a una experiencia regional de responsabilidad social empresarial en el suroccidente colombiano

\section{Actores del proceso}

En el desarrollo de la Región Socialmente Responsable del Norte del Cauca se identifican los siguientes actores: Algunos empresarios de la zona, quienes fueron los propulsores de la iniciativa con el objetivo de realizar alianzas que les permitieran aportar al desarrollo de la región; los actores gubernamentales (alcaldes de los municipios involucrados en el proyecto, el Departamento Nacional de Planeación y el Departamento de Prosperidad Social) quienes aportaron fundamentalmente en la fase de diagnóstico y en el compromiso en sus planes de gobierno con otros actores para el desarrollo de la región; y los actores del sector educativo (entidades de formación a nivel técnico, tecnológico, profesional - Comfacauca) quienes aportaron en la creación de condiciones para mejorar el nivel de formación de las personas de la región, que les permitieran integrarse al sector productivo. Los empresarios aportan en la financiación y ejecución de los proyectos para la estrategia. En mucho casos se plantean proyectos cofinanciados y operados con otros actores tanto públicos como privados.

\section{Principales líneas de actuación}

A partir de la integración de los actores anteriormente mencionados, se plantea como objetivo para el mejoramiento de la región identificar cuales serían las principales líneas de intervención de acuerdo con las capacidades de los actores y los recursos disponibles. En asocio con el Departamento de Prosperidad Social, los empresarios de la zona franca del Cauca y algunas de sus fundaciones, establecieron la denominada Alianza por lo Social, e identificándose 5 líneas de intervención con 14 proyectos orientados principalmente al municipio de Puerto Tejada, que se presentan en la tabla No. 3. 
Tabla No. 3 Líneas de Intervención de la Estrategia regional de RSE

\begin{tabular}{|c|c|c|}
\hline $\begin{array}{l}\text { Línea de } \\
\text { Intervención }\end{array}$ & Proyecto & Descripción \\
\hline \multirow{2}{*}{$\begin{array}{l}\text { Atención } \\
\text { Básica }\end{array}$} & $\begin{array}{l}\text { Aseguramien- } \\
\text { to en Salud }\end{array}$ & $\begin{array}{l}\text { Jornadas de aseguramiento en Salud para la población de } \\
\text { los } 11 \text { barrios priorizados de Puerto Tejada }\end{array}$ \\
\hline & Identificación & $\begin{array}{l}\text { Jornadas de identificación para la población de los } 11 \\
\text { barrios priorizados de Puerto Tejada }\end{array}$ \\
\hline \multirow{5}{*}{$\begin{array}{l}\text { Desarrollo } \\
\text { Social }\end{array}$} & $\begin{array}{l}\text { Generaciones } \\
\text { con Bienestar }\end{array}$ & $\begin{array}{l}\text { Promueve la garantía de los derechos y previene su vulnera- } \\
\text { ción. Gestiona la activación de las rutas de restablecimiento } \\
\text { de derechos a partir del empoderamiento de los niños, ni- } \\
\text { ñas y adolescentes y la promoción de la corresponsabilidad } \\
\text { con la familia y la sociedad. } \\
\text { Contribuye a la prevención de reclutamiento ilícito, del } \\
\text { trabajo infantil, del embarazo adolescente, del uso y abuso } \\
\text { de sustancias psicoactivas, y de la violencia intrafamiliar }\end{array}$ \\
\hline & $\begin{array}{l}\text { Música para } \\
\text { la Reconcilia- } \\
\text { ción }\end{array}$ & $\begin{array}{l}\text { Ofrece formación artística musical a niños, niñas, adoles- } \\
\text { centes y jóvenes entre los } 6 \text { y } 25 \text { años como parte de la } \\
\text { Atención Psicosocial Integral, prioritariamente a las víctimas } \\
\text { de infracciones al Derecho Internacional Humanitario o vio- } \\
\text { laciones graves y manifiestas a las normas internacionales } \\
\text { de Derechos Humanos, ocurridos con ocasión del conflicto } \\
\text { armado interno y a la población más vulnerable. }\end{array}$ \\
\hline & $\operatorname{ReSA}$ & $\begin{array}{l}\text { Busca mejorar el acceso y el consumo de los alimentos de } \\
\text { las familias rurales mediante la implementación de huertas } \\
\text { hacia la producción de alimentos para el autoconsumo que } \\
\text { incentive el ahorro por la vía del no gasto, la utilización } \\
\text { de alimentos y productos locales y el fomento de hábitos } \\
\text { alimentarios saludables }\end{array}$ \\
\hline & $\begin{array}{l}\text { Jóvenes a lo } \\
\text { bien }\end{array}$ & $\begin{array}{l}\text { Es la alianza estratégica entre agencias internacionales, } \\
\text { SENA, autoridades locales y de policía, con el fin de } \\
\text { ofrecer a jóvenes y familias vulnerables, formación pro- } \\
\text { fesional e integral en sus barrios que les permita tener } \\
\text { una vida útil a la sociedad. }\end{array}$ \\
\hline & $\begin{array}{l}\text { Familias con } \\
\text { Bienestar }\end{array}$ & $\begin{array}{l}\text { Busca mejorar la convivencia familiar. Promueve hábitos de } \\
\text { vida saludable, optimiza las condiciones de vivienda de las } \\
\text { familias y desarrolla acciones para prevenir y proteger los } \\
\text { derechos de los niños y niñas menores de } 18 \text { años. }\end{array}$ \\
\hline $\begin{array}{l}\text { Educación y } \\
\text { generación } \\
\text { de Ingresos }\end{array}$ & $\begin{array}{l}\text { Jóvenes en } \\
\text { Acción }\end{array}$ & $\begin{array}{l}\text { Programa del Gobierno Nacional de transferencias mo- } \\
\text { netarias condicionadas hacia la formación para el trabajo, } \\
\text { la generación de ingresos autónomos y el mejoramiento } \\
\text { de las condiciones de vida de los jóvenes en condición de } \\
\text { pobreza. }\end{array}$ \\
\hline
\end{tabular}


Andrés Ramiro Azuero-Rodríguez, Mónica García-Solarte, Benjamín Betancourt-Guerrero Aproximación a una experiencia regional de responsabilidad social empresarial en el suroccidente colombiano

\begin{tabular}{|c|c|c|}
\hline $\begin{array}{l}\text { Línea de } \\
\text { Intervención }\end{array}$ & Proyecto & Descripción \\
\hline & $\begin{array}{l}\text { Incentivo } \\
\text { para la } \\
\text { Capacitación y } \\
\text { el Empleo } \\
\text {-ICE- }\end{array}$ & $\begin{array}{l}\text { Beneficia a personas en situación de pobreza extrema o } \\
\text { desplazamiento, a través de un modelo flexible de forma- } \\
\text { ción de capacidades que articula la oferta educativa y el } \\
\text { sector privado para permitir una efectiva inserción en el } \\
\text { mercado laboral. }\end{array}$ \\
\hline & $\begin{array}{l}\text { Capitalización } \\
\text { Micro- } \\
\text { empresarial }\end{array}$ & $\begin{array}{l}\text { Cofinancia procesos asociativos que generen valor agre- } \\
\text { gado a productos primarios, al tiempo que promueve el } \\
\text { acceso a mercados, y la vinculación formal de personas en } \\
\text { edad de trabajar, así como impulsa la modernización de los } \\
\text { sistemas de producción para promover la capitalización de } \\
\text { bienes de uso social y la generación de empleo. }\end{array}$ \\
\hline Habitabilidad & $\begin{array}{l}\text { Mejoramiento } \\
\text { de vivienda }\end{array}$ & $\begin{array}{l}\text { Promueve el asentamiento de la población a través de } \\
\text { acciones de impacto rápido en la intervención de sus ne- } \\
\text { cesidades básicas habitacionales. }\end{array}$ \\
\hline \multirow{3}{*}{$\begin{array}{l}\text { Desarrollo } \\
\text { Comunitario }\end{array}$} & $\begin{array}{l}\text { Espacios } \\
\text { Deportivos }\end{array}$ & $\begin{array}{l}\text { Construcción de las dos cubiertas e iluminación del estadio } \\
\text { de la Villa Olímpica del municipio de Puerto Tejada }\end{array}$ \\
\hline & $\begin{array}{l}\text { Legión del } \\
\text { Afecto }\end{array}$ & $\begin{array}{l}\text { Generar espacios para la inclusión social y la reconciliación } \\
\text { de poblaciones vulnerables y víctimas de la violencia en } \\
\text { situación de riesgo y marginalidad a través de la imple- } \\
\text { mentación de metodologías comunitarias de acompaña- } \\
\text { miento socialmente masivo que contribuyan a visibilizar } \\
\text { y promover las capacidades de liderazgo y participación } \\
\text { cívica, reconstruir sus proyectos de vida y el tejido social, } \\
\text { al diseño de oportunidades dignas y legales ajustadas al } \\
\text { contexto de sus entornos de vida, para mejorar la con- } \\
\text { vivencia, la construcción de confianza y deslegitimar las } \\
\text { alternativas de violencia e ilegalidad en estas poblaciones } \\
\text { y comunidades en alto riesgo. }\end{array}$ \\
\hline & $\begin{array}{l}\text { Bloqueras } \\
\text { Solidarias }\end{array}$ & $\begin{array}{l}\text { Programa de encadenamientos productivos cuyo objetivo } \\
\text { es contribuir a erradicar la pobreza extrema en Colombia, } \\
\text { ofreciendo una solución social innovadora para que las } \\
\text { familias mejoren o construyan sus viviendas con bloques } \\
\text { de concreto que ellos mismos producen. La mitad de los } \\
\text { bloques de concreto producidos son propiedad de las } \\
\text { familias beneficiarias para que puedan ser utilizados en la } \\
\text { mejora o construcción de sus viviendas. El otro } 50 \% \text { queda } \\
\text { a disposición del proyecto para ser comercializadoy de esta } \\
\text { manera garantizar el sostenimiento del programa. }\end{array}$ \\
\hline
\end{tabular}

Fuente: Datos institucionales de la zona franca del Cauca. 
Para identificar los principales avances, logros, aprendizajes, fallas y retos derivados de la estrategia Región Socialmente Responsable Norte del Cauca, se llevaron a cabo entrevistas individuales (estructuradas, semi-estructuradas o abiertas), grupos focales y talleres de formulación colectiva de las lecciones aprendidas, con los actores que se encontraban relaionados de manera directa o indirecta con la estrategia. A partir de ahí se identifican:

\section{Logros, avances, aprendizajes, dificultades y retos del proceso}

En la consulta realizada a los diversos actores que se encuentran relacionados directamente o indirectamente con el proceso, se identificaron los siguientes elementos:

\section{Logros}

- Poder articular a diversos actores como empresarios, gobiernos locales, comunidad e instituciones en torno a una experiencia de construcción de región desde la perspectiva de múltiples intereses.

- Fortalecimiento de la capacidad de formación en la región con la consolidación de la presencia de instituciones de formación técnica, tecnológica y profesional. Se reconoce especialmente la presencia de Unicomfacauca y otras ofertas de la Universidad del Valle y del Cauca, entre otras.

- Proyectos articulados para la región que incluye atención a temas prioritarios de grupos indígenas, como el Caso del proyecto Agua para todos, cofinanciado y operado entre Mexichem, Asocaña, UNICEF, Corpopalo, ANDI. Igualmente, proyectos relacionados con la protección de la cosmovisión indígena y capacitación en buenas prácticas agropecuarias y sensibilización ambiental.

- Involucramiento del gobierno nacional a través del DNP y el DPS en la formulación de proyectos conjuntos y cofinanciación para generar un alto impacto en la región.

- Inclusión de la propuesta del acueducto regional para el Norte del Cauca. En el marco de la estrategia se propuso el diseño apoyado de estudios técnicos que sirvieron de base para elaborar el proyecto que se ejecuta actualmente que incluye cambios a la propuesta original.

- Participación es espacios de discusión de la región como los foros del proyecto "proyección milenio" del Programa de las Naciones Unidas para el Desarrollo. La estrategia Región Socialmente Responsable se presentó en diversas oportunidades, se resalta la realizada en el marco del Foro-Taller "Región y gobernabilidad en municipios nortecaucanos con población significativamente afrodescendiente" donde participaron candidatos a los gobiernos locales de la zona. 
- Apuesta por la recuperación del tejido social con la resocialización de Jóvenes en alto riesgo a través de los programas en asocio con la policía nacional, el SENA, y otras instituciones y empresas.

- Aportes en la visión para la inversión en espacios recreativos en la zona. La caja de compensación del Cauca Comfacauca construyó el Parque Recreativo las Ceibas como una alternativa para el esparciemiento en la zona y plantea como área de influencia de las zonas rurales y urbanas de los municipios de Buenos Aires, Villarrica, Caloto, Corinto, Padilla, Miranda, Guachené y Santander de Quilichao.

- El desarrollo de la estrategia permite una visibilización de la región del Norte del Cauca en el ámbito nacional e internacional, por ser objeto de estudio en ponencias, presentaciones, intervenciones institucionales tanto del sector público como privado.

\section{Avances}

- Aumento del número de contratación con personas de la región, con el avance en el empleo local que generan las empresas ubicadas en la zona.

- Vinculación del contrato Plan para la región como una oportunidad de participar en las discusiones sobre las inversiones del gobierno en la zona.

- Generación de vínculos de confianza con los grupos indígenas y afrodescendientes por la oportunidad de abrir algunos espacios para el diálogo y la discusión sobre temas de región.

- Fortalecimiento de las dinámicas que ya venían en proceso en la región con la creación de pymes y el desarrollo de negocios inclusivos con actores locales.

- Inclusión de la dimensión rural en las discusiones y apoyo a proyectos como el de vivienda rural en algunas zonas de la región.

- Aspecto de mejora en las inversiones en el sector Salud por la visibilización de la problemas de la zona. Se plantea como ejemplo la consolidación del Hospital del municipio de Villarica.

\section{Aprendizajes}

- Importancia de la creación de espacios de encuentro y diálogo alrededor de temas comunes de región, permitiendo articular en proyectos conjuntos focalizados a actores con diferentes intereses y perspectivas.

- Las problemáticas de una región no pueden ser abordadas desde las capacidades y perspectivas de un solo actor. El concurso de diversos actores en torno a proyectos concertados tiene una mayor probabilidad de alto impacto en los resultados.

- Todos los actores tanto empresariales, gobierno, instituciones, organizaciones de todo tipo, tienen diferentes capacidades para abordar la solución y ejecución 
de proyectos, por lo que aprovechando dichas capacidades en forma articulada se pueden lograr mayores efectos en la región.

- Creación de una línea de base de conocimientos y conceptos compartidos para la discusión a través de la Capacitación de formadores. De esta manera el diálogo se hace sobre ideas base en común.

- Aprovechando el desarrollo de experiencias en la región, un aprendizaje planteado radica en la necesidad de realizar un proceso sistemático de empoderamiento y liderazgo de los dirigentes de turno en los gobiernos locales que permita la articulación, propuesta y ejecución de proyectos conjuntos.

\section{Dificultades}

- Retrasos en la ejecución de algunas alianzas derivados de algunos problemas institucionales o trámites administrativos.

- Falta de liderazgo de algunos actores durante todo el proceso. Se plantea la necesidad de participación a largo y mediano plazo.

- Falta de reconocimiento de algunos de los actores involucrados en algunos espacios.

- Mayor disponibilidad de los recursos del Gobierno nacional (DPS) para la ejecución de algunos proyectos en la región.

- Falta de sistematización y difusión de los logros de la estrategia para hacerla mas visible.

- El no compartir los diagnósticos y procesos que se están realizando individualmente por algunos actores para que no se pierdan los procesos y las experiencias y evitar que se creen reprocesos.

- Centralizar la dirección del proceso en pocas personas.

- La velocidad con la que venía el proceso cambió de ritmo con la necesidad de cumplir ciertos procesos administrativos de los diferentes actores, en algunos casos con mayor énfasis en el sector público.

- La falta de continuidad del proceso en los gobiernos locales pues depende del alcalde de turno.

- Los actores plantean que una situación a corregir es el ampliar el alcance de acción de la estrategia a otros municipios.

\section{Retos}

- Articulación de todos los actores del territorio (indígena-afro-campesino-gobierno-academia y empresa) y conciliación de sus diferentes intereses y visiones de futuro sobre la región. 
Andrés Ramiro Azuero-Rodríguez, Mónica García-Solarte, Benjamín Betancourt-Guerrero

Aproximación a una experiencia regional de responsabilidad social empresarial en el suroccidente colombiano

- Flujo de recursos económicos del gobierno nacional para mantener los proyectos planteados.

- Preparación de todos los actores sobre diferentes alternativas frente al escenario del Postconflicto en una región con diversos problemas asociados al conflicto.

- Los cambios generacionales y los estilos de liderazgo que permitan seguir construyendo en temas comunes en la región. Ello implica generar siempre nuevos espacios para el empoderamiento de los actores.

- Darle continuidad al desarrollo de la estrategia Región Socialmente Responsable en el norte del Cauca.

- Medir el impacto en los grupos de interés del desarrollo de la estrategia Región Socialmente Responsable Norte del Cauca.

- Compromiso de los gobiernos locales de turno para dar continuidad en sus planes de desarrollo a proyectos de región articulados con otros actores.

- Recuperación del proyecto de acueducto regional.

- Liderazgo de la academia para la difusión y recolección de los proyectos y logros de la comunidad y los actores regionales.

- Pertinencia de la oferta y calidad de los programas de formación del sector académico.

- Aprovechar la situación actual del país para mejorar las condiciones del norte del Cauca.

\section{Conclusiones}

A pesar de que los actores consultados plantean que los problemas de la región continúan siendo los mismos encontrados en los diagnósticos iniciales al comienzo de la estrategia (Educación, Saneamiento básico, Empleo, Pobreza, Inseguridad, entre otros), se destaca la importancia de lograr la articulación de actores en torno a problemas comunes de región. Se mencionan avances en educación, con mayor presencia de instituciones educativas, en el servicio de agua potable, con el proyecto del acueducto regional que se encuentra en marcha y que aunque ha sufrido transformaciones fue apalancado desde el principio por la estrategia regional de RSE. En cuanto a recreación se encuentra el desarrollo actual del mega parque las Ceibas en Puerto Tejada. De acuerdo con los actores se ha mejorado el nivel de empleo de los trabajadores locales en las empresas de la zona franca, a pesar de que muchas se han retirado de la región según la Cámara de Comercio.

Aunque desde sus inicios la estrategia Región Socialmente Responsable busca lograr la articulación de los actores para el buen éxito de los proyectos propuestos, se plantea sin embargo que aún son incipientes algunos acuerdos institucionales, la presencia del gobierno departamental y nacional en algunos casos ha dificultado la consecución y disposición de los recursos por diversos trámites de tipo 
administrativo, dejando parte de la ejecución de proyectos en la iniciativa de los empresarios, como han venido haciendo desde el inicio de la estrategia.

Muchos actores perciben que la estrategia inicial de responsabilidad social del norte del Cauca fue limitada en cuanto se diseñó como un estudio piloto en cuatro municipios del norte del Cauca, en donde se asientan las principales empresas que lideraron el proceso inicial. Lo anterior implicaría involucrar a más actores y ampliar el ámbito de los proyectos a otros municipios del norte del Cauca, siempre y cuando se cuente con el respaldo y compromiso de los actores de las nuevas zonas que quieren ingresar a la estrategia.

Por aspectos territoriales y muchas veces culturales, muchos de los actores del norte del Cauca se consideran más cercanos del Valle del Cauca por la misma configuración del Valle geográfico del río Cauca. Se plantea que en algunos casos, los habitantes conocen Cali, pero no Popayán que es la capital del Departamento, lo que implica que la visión de región se debe realizar en un contexto mucho más amplio al meramente departamental, siendo un espacio importante de convergencia para la construcción de región.

Considerando que todavía persisten muchos de los problemas de tipo social, económico y ambiental que ha tenido la región históricamente, desde una de las perspectivas que pueda aportar al desarrollo local, una estrategia regional de RSE como la del norte del Cauca, se debe consolidar aún más para dar continuidad y posibilidades a nuevos proyectos en conjunto e individuales que transformen la realidad de los actores en torno a la esfera de influencia de los mismos.

\section{Recomendaciones}

Frente a la situación planteada por los actores consultados de ampliar el margen de acción e incorporar más participantes en la estrategia Región Socialmente Responsable Norte del Cauca, surge como recomendación la propuesta de la creación de una instancia de diálogo y encuentro entre los actores de la región. Una posibilidad que ha tenido éxito en otros lugares del mundo, es la creación de un concejo regional de responsabilidad social, involucrando diferentes actores sociales.

Para poder identificar los logros de la estrategia en términos de la perspectiva de los beneficiarios, se podrían realizar estudios de evaluación de impactos de los proyectos desarrollados en el marco de la estrategia Región Socialmente Responsable.

Igualmente, es importante sostener el proceso ya iniciado en otras etapas en materia de sensibilización y formación tanto en temas de gobierno como de responsabilidad social y desarrollo local para la región. Por ello, en el marco del desarrollo del proyecto a la región se le entregará el diseño de un diplomado en Desarrollo Local. 


\section{BIBLIOGRAFÍA}

1. ALBURQUERQUE, F. (2003). Teoría y práctica del enfoque del desarrollo local. Instituto de Economía y Geografía. Consejo Superior de Investigaciones Científicas, Madrid.

2. ALOSON, J. (2006). 10 años de la ley Páez. Impacto económico. Universidad ICESI

3. ALOSON, J. y LOTERO, A. (2006). 10 años de la ley Páez. Transformación de la economía caucana. Universidad Icesi.

4. AZUERO R. A., MELO, J. M. y GARCÍA S., M. (2011). Una presentación de la región socialmente responsable del Norte del Cauca. Cuadernos de Administración, 27(45), 109-121.

5. BARROSO T., F. G. (2008). La responsabilidad social empresarial: un estudio en cuarenta empresas de la ciudad de Mérida, Yucatán. Contaduría y administración (226), 73-91.

6. BOWEN, H.R. (1953). Social Responsibilities of the Businessman. New York, NY: Harper \& Row.

7. CARROLL, A. B. (1999). Corporate social responsibility evolution of a definitional construct. Business \& society, 38(3), 268-295.

8. CENTRO DE INVESTIGACIONES Y DOCUMENTACIÓN SOCIOECONÓMICA - CIDSE. (2009). Desigualdades étnico raciales, acción colectiva, etnicidad y resistencia en el norte del Cauca y sur del Valle. Universidad del Valle. Cali, Colombia.

9. CRESPO R., F. (2010). Entre el concepto y la práctica: responsabilidad social empresarial. Estudios Gerenciales, 26(117), 119-130.

10. DRUCKER, P.F. (1984). The New Meaning or Corporate Social Responsibility. California Management Review, 26(2), 53-63.

11. ELIZALDE H., A. (2003). Planificación Estratégica Territorial y Políticas Públicas para el Desarrollo Local. Instituto Latinoamericano y del Caribe de Planificación Económica y Social (ILPES), Serie gestión pública 29. Chile

12. FAJARDO, E. (2010). Responsabilidad social empresarial: una reflexión desde la gestión; lo social y ambiental. Bogotá: Universidad Nacional de Colombia, Facultad de Ciencias Económicas

13. FLÓREZ D. R., NEIRA, R. \& OSORIO M. (2005). El problemático proceso de construcción de región, Los proyectos de regionalización en la zona centro occidental de Colombia. Revista Universidad de Caldas, 25 (2), 83-106.

14. FRIEDMAN, M. (1970). The Social Responsibility of business is to Increase its profits. The New York Times Magazine.

15. FUNDACIÓN CARVAJAL (2007). Estudio de caracterización social y económico de los trabajadores de PAVCO y su entorno inmediato. Guachené - Cauca.

16. GARCÍA, A. (2007). Cultura Organizacional y cultura afrocolombiana. Un estudio cualitativo de caso en la planta de Pavco en Guachené, Cauca. Tesis de Maestría en Administración. Universidad del Valle.

17. GARRIGA, E. and MELÉ, D. (2004). “Corporate Social Responsibility theories: mapping the territory”. Journal of business ethics, 53, 1-2, pp 51-71.

18. GIRALDO H., G. (2011). Responsabilidad social empresarial en Antioquia. Revista Universidad EAFIT, 44(149), 38-59.

19. GUERRA S., A. (2007). De la responsabilidad social empresarial, a la ética en el cambio organizacional. Compendium: revista de investigación científica, (18), 77-90. 
20. GUTIÉRREZ, R., AVELLA, L. F., y VILLAR, R. (2006). Aportes y desafíos de la responsabilidad social empresarial en Colombia. Fundación Carvajal.

21. INTERNATIONAL ORGANIZATION OF STANDARIZATION - ISO 26000 (2010). Guidance on Social Responsibility. Committee Draft ISO /CD 26000. ISO/TMB/WG SR N 157. Chapters 2. Ginebra, Suiza: ISO.

22. MARK-HERBERT, C. y VON SCHANTZ, C. (2007). Communicating Corporate Social Responsibility - Brand management. Electronic Journal of Business Ethics and Organization Studies, 12(2), 4-11.

23. MAYAN, M. J. (2009). Essentials of Qualitative Inquiry. California: Left Coast Press.

24. MÉNDEZ, C. E. (2006). Metodología. Diseño y desarrollo del proceso de Investigación con énfasis en ciencias empresariales. Bogotá: Limusa.

25. MERCADO S., P., y GARCÍA H., P. (2007). La responsabilidad social en empresas del Valle de Toluca (México): un estudio exploratorio. Estudios Gerenciales, 23(102), 119-135.

26. MONTES, J. (2008). Ecoeficiencia: una propuesta de responsabilidad ambiental empresarial para el sector financiero colombiano (Tesis de Maestría). Facultad de Minas. Tesis de Grado para optar al título de Maestría en Medio Ambiente y Desarrollo. Universidad Nacional de Colombia. Medellín, Colombia.

27. PAMPLONA S., M. J. (2014). La nueva energía paisa: una región como estrategia de desarrollo local en Antioquia, Colombia. Analecta Política, 4(7), 369-389.

28. PROGRAMA DE LAS NACIONES UNIDAS PARA EL DESARROLLO PNUD (2009). Una apuesta para avanzar hacia el logro de los Objetivos de Desarrollo del Milenio en municipios del Norte del Cauca con población significativamente afrodescendiente. Colombia.

29. PORTER, M. y KRAMER, M. (2002). The Competitive Advantage of the Corporate Philanthropy. The Harvard Business Review.

30. RAUFFLET, E. (2011). Responsabilidad corporativa y desarrollo sostenible: una perspectiva histórica y conceptual. Cuadernos de Administración, 26(43), 23-32.

31. ROA, M.G. (2000) Procesos de interacción laboral con trabajadores provenientes de comunidades tradicionales: estudios de caso en la región Paez. Trabajo de grado Maestría en Administración. Universidad Icesi.

32. ROSALES R. y CHAUCA, P. (2010). Desarrollo local: teorías, políticas y experiencias. Madrid: Plaza y Valdés Editores.

33. SALINAS, L.E. (2008). Conceptos claves. Región Socialmente Responsable. Documento presentado en el encuentro nacional de regiones responsables de Colombia. Paipa - Boyacá.

34. STEINER, G.A. (1971). Business and Society. New York, NY: Random House.

35. TECCO, C. A., y LUCCA, C. M. (1998). Fortalecimiento institucional y desarrollo local. La asistencia técnica a los municipios en un contexto de crisis y cambios, 23.

36. VANTULDER R., y VAN DER ZWART, A. (2006)."International business-society management linking corporate responsibility and globalization". London and New York: Routledge.

37. VILLACORTA, A. E. (2008). Microrregiones y mancomunidades municipales: nuevas formas de gestión del territorio. Prisma, (22), 149-166.

38. VILLAR, A. (2004). Una década de desarrollo local en Argentina. Balance y perspectivas. Revista Mundo Urbano No. 24.

39. WARTICK, S. L., \& MAHON, J. F. (1994). Toward a Substantive Definition of the Corporate Issue Construct A Review and Synthesis of the Literature. Business \& Society, 33(3), 293-311.

40. WILLIG, C. (2001). Introducing qualitative research in psychology. Adventures in theory and method. New York: Open University Press. 